\title{
siRNA-based nanoparticles for cancer therapy: hurdles and hopes
}

\begin{abstract}
In recent times, profound advances in therapeutic strategies for cancer therapy have been made. Most of these strategies including cationic polymers, small molecule inhibitors and monoclonal antibodies originating from the bench have resulted in successful translations to the bedside. One of these remarkable strategies was the potential of small interfering RNA (siRNA) to regulate gene expression by RNA interference (RNAi). An avalanche of research has indeed proven that RNAi is extremely essential in cancer therapeutics, as well as a myriad of diseases. Their potential as therapeutic platforms however carry some limitations including delivery challenges, potential side effects, disturbance of intrinsic gene silencing mechanisms, and induction of the innate immune response. Some attempts have been made to overcome these challenges in order to improve the efficacy of RNAi-based therapeutics. Nanostructured entities (nanoparticles) and their formulations provide immense opportunities to substantially alter the treatment regimens, i.e. improving drug bioavailability, improving tissue permeability, and improving payload integrity. The objectives of this article are to (a) explain siRNA mechanism in the context of cancer therapy; (b) highlight the usefulness of nanoparticles-based delivery, and (c) review the prospects and challenges of the ongoing siRNA-based clinical research.
\end{abstract}

Keywords: no particles, siRNA, cancer therapy, delivery strategies, clinical trials
Volume 4 Issue 6 - 2016

Stanley Moffatt

Regent University College of Science and Technology, Ghana

Correspondence: Stanley Moffatt, School of Informatics, Engineering and Technology, Regent University College of Science and Technology,Accra, Ghana, Tel +233242169839, Email Stanley.moffatt@regentghana.net

Received: November 24, 2016 | Published: December 29, 2016

\section{Introduction}

RNA has generally been associated with two fundamental functions. Single-stranded messenger RNA (mRNA) was considered to be a passive transmitter of information between DNA and protein synthesis. The catalytic role of RNA was highlighted in 1986 where ribosomal RNA (rRNA) and transfer RNA (tRNA) were discovered to code for information during protein synthesis.

In 1998, Fire et al., ${ }^{1}$ discovered the ability of double-stranded RNA (dsRNA) to silence gene expression in Caenorhabditis elegans. In 2001, Elbashir et al., ${ }^{2}$ demonstrated that synthetic small interfering RNA (siRNA) could knock down a gene in a sequencespecific manner. Further, he in vivo therapeutic potential of siRNA was demonstrated in transgenic mice with a sequence from hepatitis C virus. ${ }^{3}$ Several others ${ }^{4,5}$ have shown tremendous interest in siRNA technology in cancer therapy in particular. RNAi is one of the fastest advancing fields in biology. In 2004, the technology entered a Phase I clinical trials in humans for wet age-related macular degeneration. ${ }^{6,7}$ In 2010, the first-in-human Phase I clinical trial was started, ${ }^{8}$ against solid tumors using a targeted nanoparticle delivery system.

The promise of siRNA technology calls for a review to address

Table I Naked siRNA applications: causes and challenges fully the strengths and weaknesses of their applications, in an effort to highlight solutions that could transform siRNA technology into a conventional treatment against various malignancies. Besides the overview, the possible uses, challenges, and the various strategies for siRNA delivery, we would highlight some of the successful in vivo in humans. Finally, an evaluation of the future prospects of the technology in cancer treatment is discussed.

\section{siRNA in the clinic}

\section{Difficulties in therapeutic application}

As indicated, siRNAs hold great promise as therapeutic agents, however, effective in vivo delivery has been challenging to use in the clinic (Table 1). ${ }^{9}$ In fact, "naked" siRNA is susceptible to several obstacles that reduce their therapeutic efficacy. ${ }^{10}$ Furthermore, due to their size and highly polyanionic nature ${ }^{10}$ unmodified siRNA molecules cannot readily enter the cells. Even after entering the cells, repeated dosing is required since their effects are diluted at each cell division." "Naked" siRNA molecules have also shown to be toxic which is achieved via three main mechanisms, i.e, saturation, competition and stimulation of the immune response. View it in a separate window.

\begin{tabular}{lll}
\hline Challenges & Cause & Solution \\
\hline & Nuclease susceptibility & Topical or local administration \\
Short half life & Quick renal clearance & $\begin{array}{l}\text { Functional modification of the sugars or the bases } \\
\text { of oligo-ribonucleotides }\end{array}$ \\
& Phagocyte uptake & Encapsulation in nanoparticles \\
Reduced systemic uptake & Anionic nature & Conjugation with nanoparticles \\
& Size too large to permeate membrane & \\
\hline
\end{tabular}


Table Continued....

\begin{tabular}{lll}
\hline Challenges & Cause & Solution \\
\hline $\begin{array}{l}\text { Transient effect } \\
\text { Toxic effect }\end{array}$ & Dilution of siRNA concentration & shRNA for genome integration \\
Saturation of siRNA & Reduced accessibility to miRNA & Optimal dosing for targeting \\
Stimulation of immune & $\begin{array}{l}\text { Toll-Like Receptor (TLR) activation and type I } \\
\text { (Interferon) IFN response }\end{array}$ & Methylation of 2'OH in nanoparticles \\
Off-target effect & Non-TLR mediated immune response & Methylation of 2'OH in nanoparticles \\
\hline
\end{tabular}

Saturation of RNAi machinery and competition in miRNA pathway

The introduction of exogenous dsRNA into the cell could perturb the RNAi in the cell. Consequently, the this could impede the cellular machinery recruited for gene silencing, making it less accessible to miRNAs entering the natural cellular pathway. ${ }^{10,12} \mathrm{~A}$ number of research results all point to the essence of intact RNAi machinery for mammalian cells, as evidenced by the embryonic lethality of Dicer knockouts.

\section{Stimulation of immune response}

Some of the siRNA sequences could stimulate innate immune response. Briefly, when nucleic acids enter lysosomal or endosomal pockets, TLR-3, 7, and 8 are sensitized to activate interferon-alpha and inflammatory cytokines via nuclear factor k-light chain enhancer of activated $\mathrm{B}$ cells. ${ }^{13}$ The innate immune response that bypasses TLR activation is triggered by cytoplasmic RNA sensors, including dsRNA-binding protein kinase. This pathway can further activate interferon-beta and other inflammatory mediators.

\section{siRNA delivery strategies: then and now}

siRNA therapeutics were first delivered locally using naked or chemically modified forms. ${ }^{14}$ However, the complexity of various physiological interactions has signaled the necessity to protect gene material in order to ensure its target specificity. Preliminary approaches to protect siRNA arose from DNA transfection techniques involving mechanical (e.g. sonoporation and electroporation), as well as chemical and biological approaches (e.g. viral vectors).

Nanotechnology has been a most promising and surprising "science on the block" in current molecular medicine because the huge impact of nano-therapeutics in terms of efficacy s enormous. With the avalanche of research using nanoparticles, exciting possibilities and workable outcomes may resolve most of the current challenges in drug delivery. This is also likely to remove the hurdles to discovering the "magic bullet" for cancer, by changing unprofitable treatment regimens. With the advent of nanotechnology, a number of nanoparticle therapeutics has been made commercially available. The first is the use of liposome as a drug delivery system, because of the peculiarity of their inner structure which can facilitate drug transport to a targeted site. Furthermore, modifications with poly(ethylene) glycol (PEG), have increased their bioavailability. Nevertheless, limiting factors concerning their stability and transportation efficiency still remain.

\section{Nanoparticle-siRNA delivery challenges}

Nanoparticle-based drug delivery still has some challenges. First, the vehicles must cross the biological barriers in the mucosa as well as the cellular and humoral components of the immune system, in order to reach the target cells. The particles are also sequestered by negatively charged blood serum proteins. Moreover, they are liable to opsonization by immune cells. Even though nanostructures generally undergo rapid clearance, the reticuloendothelial system is able to trap and degrade intravenously injected particles $>100 \mathrm{~nm}$ in diameter, by activated monocytes and macrophages. On the other hand, particles $<4 \mathrm{~nm}$ in diameter usually are able to undergo rapid renal clearance. With the foregoing, the optimal diameter of nanoparticles for intra-tumoral delivery, avoidance of renal clearance, and the reticuloendothelial system, should be between $5-100 \mathrm{~nm}$.

In the light of these limitations, however, nanoparticles can be surface-functionalized or size-engineered to overcome these barriers. Indeed, surface functionalization with PEG or other hydrophilic conjugates can provide steric stabilization, help in evading the immune system, and render protection against the harmful effects of the surrounding micro-environment.

The enhanced permeability and retention effect of malignant cancers and tumors can also be exploited for drug delivery. The ill-formed tumor blood vessels retain molecules of aparticular size to a greater extent than in healthy ones. This phenomenon allows therapeutic nanoparticles to accumulate in target tissues, thereby allowing for controlled drug release over time, as opposed to drugs prepared by conventional means. Limitations can however arise in the microenvironment and interfere with achieving therapeutic doses of these molecules. An intervention could include normalizing the tumor vasculature (eg, with antiangiogenic therapy). Once inside the target cells, the nanoparticulate RNAi must escape the endosomal enzymes in the lysosome to avoid degradation. Fusogenic peptides and lipids, $\mathrm{pH}$-sensitive polyplexes and lipoplexes, have been used to improve endosomal escape. ${ }^{15}$

Research indicates that nanoparticles have systemic toxicity, mostly in the liver.$^{16}$ Furthermore, frequent injections of siRNA using current methods could pose a limitation in their clinical application due to decreased patient compliance. ${ }^{17,18}$ Again, the heterogeneity of tumors could also induce the development of inherent resistance to some RNAi, owing to factors such as somatic mutations, and germline single nucleotide polymorphisms. ${ }^{9}$

\section{Conclusions and future prospects}

siRNA technology has remarkably progressed from mere academic discovery to a potential class of treatment modality against human diseases. Early excitement about the full exploitation of siRNA technology was however soon dampened by hurdles such as (a) identifying strategies to select potent target-specific siRNA, (b) drastically reducing immune stimulation (c) drastically reducing competition with cellular RNAi, and (4) remarkably improving targetspecific in vivo delivery. At the moment, many of these issues are being addressed. 
The advent of nanomedicine has represented a milestone, contributing strongly towards a solution to problems often encountered in the clinical setting. However, their delivery remains one of the daunting challenges to the use of siRNA therapeutics in the clinical setting. To this end, several strategies aimed at mitigating this limitation are being explored. Looking forward, synthetic nanoparticles will likely have a fundamental role in the systemic.

Even though tremendous progress has been made in the use of siRNA-based nanoparticles in cancer therapeutics, the ability to: overcome systemic biological barriers, differentiate cancerous from normal tissues, and deliver therapeutic doses of siRNA, are still the key indicators of the success of siRNA-based therapy. It is also imperative for researchers to be mindful of patient compliance when considering repeated dosing. Again, the polygenic nature of cancer calls for a combinatorial approach of siRNA with biotherapy, radiotherapy, chemotherapy, and/or immunotherapy. On the whole, it is expected that siRNA-based nanotherapeutics will be the forerunner in the pool of strategies for drug development for cancer therapy.

\section{Acknowledgements}

None.

\section{Conflict of interest}

The author declares no conflict of interest.

\section{References}

1. Chen Y, Cheng G, Mahato RI. RNAi for treating hepatitis B viral infection. Pharm Res. 2008;25(1):72-86.

2. Elbashir SM, Harborth J, Lendeckel W, et al. Duplexes of 21-nucleotide RNAs mediate RNA interference in cultured mammalian cells. Nature. 2001;411:494-498.

3. McCaffrey AP, Meuse L, Pham TT, et al. Gene expression: RNA interference in adult mice. Nature. 2002;418:38-39.

4. Wall NR, Shi Y. Small RNA: can RNA interference be exploited for therapy? Lancet. 2003;362(9393):1401-1403.
5. Devi GR. siRNA-based approaches in cancer therapy. Cancer Gene Ther. 2006;13(9):819-829.

6. Whelan J. First clinical data on RNAi. Drug Discov Today. 2005;10(15):1014-1015.

7. Castanotto D, Rossi JJ. The promises and pitfalls of RNA-interferencebased therapeutics. Nature. 2009;457(7228):426-433.

8. Davis ME, Zuckerman JE, Choi CH, et al. Evidence of RNAi in humans from systemically administered siRNA via targeted nanoparticles. Nature. 2010;464(7291):1067-1070.

9. Pecot CV, Calin GA, Coleman RL, et al. RNA interference in the clinic: challenges and future directions. Nat Rev Cancer. 2011;11(1):59-67.

10. Aagaard L, Rossi JJ. RNAi therapeutics: principles, prospects and challenges. Adv Drug Deliv Rev. 2007;59(2-3):75-86.

11. Kim DH, Rossi JJ. Strategies for silencing human disease using RNA interference. Nat Rev Genet. 2007;3(3):173-184.

12. Grimm D, Streetz KL, Jopling CL, et al. Fatality in mice due to oversaturation of cellular microRNA/short hairpin RNA pathways. Nature. 2006;441(7092):537-541.

13. Kleinman ME, Yamada K, Takeda A, et al. Sequence- and targetindependent angiogenesis suppression by siRNA via TLR3. Nature. 2008;452(7187):591-597.

14. Schroeder A, Levins CG, Cortez C, et al. Lipid-based nanotherapeutics for siRNA delivery. J Intern Med. 2010;267(1):9-21.

15. Dominska M, Dykxhoorn DM. Breaking down the barriers: siRNA delivery and endosome escape. J Cell Sci. 2010;123(Pt 8):1183-1189.

16. Tanaka T, Mangala LS, Vivas-Mejia PE, et al. Sustained small interfering RNA delivery by mesoporous silicon particles. Cancer Res. 2010;70(9):3687-3696.

17. Merritt WM, Lin YG, Spannuth WA, et al. Effect of interleukin-8 gene silencing with liposome-encapsulated small interfering RNA on ovarian cancer cell growth. J Natl Cancer Inst. 2008;100(5):359-372.

18. Landen CN, Chavez-Reyes A, Bucana C, et al. Therapeutic EphA2 gene targeting in vivo using neutral liposomal small interfering RNA delivery. Cancer Res. 2005;65(15):6910-6918. 